\title{
Stent Retriever Thrombectomy Potentially Increases the Recanalization Rate, Improves Clinical Outcome, and Decreases Mortality in Acute Basilar Occlusion: A Systematic Review and Meta-Analysis
}

\author{
Liang Shu Mona Salehi Ravesh Olav Jansen Ulf Jensen-Kondering \\ Department of Radiology and Neuroradiology, University Hospital Schleswig-Holstein, \\ Campus Kiel, Kiel, Germany
}

\section{Keywords}

Basilar artery occlusion - Intravenous thrombolysis - Thrombectomy · Meta-analysis .

Systematic review

\begin{abstract}
Background: Acute basilar artery occlusion (BAO) is a devastating condition if untreated. The optimal treatment strategy, however, is unknown. Historically, interventional approaches have been favored over intravenous thrombolysis (IVT), although this is not supported by good evidence. The aim of this systematic review and meta-analysis was to summarize and compare the results for treatment modalities of BAO, namely, IVT and interventional stent retriever thrombectomy (SRT). Methods: Studies on IVT and SRT in BAO were systematically searched. Successful recanalization ( $\mathrm{TICl} \geq 2 \mathrm{~b}$ ), favorable clinical outcome (modified Rankin Scale score $\leq 2)$, mortality, and the rate of symptomatic intracranial hemorrhage $(\mathrm{SICH})$ were the target parameters. Only studies with $\geq 15$ patients were included. IVT prior to SRT was allowed. Studies were excluded (1) if $>1$ thrombectomy device was used in $>50 \%$ of the patients and (2) when data on outcome or treatment could not be parsed (e.g. registries). Odds ratios (ORs) were calculated using Mantel-Haenszel risk ratio estimation. Results are given as OR and the $95 \%$ confidence interval $(95 \% \mathrm{Cl})$. The $\chi^{2}$ test was used to compare the outcome parameters clinical outcome, recanalization, mortality, and SICH. Results: A total of 17 studies (4 on IVT and 13 on SRT) with a total of 672 patients (IVT, $n=314$; SRT, $n=358$ ) were identified. Cumulatively, we found a highly significantly improved clinical outcome (43 vs. $31 \%, p=0.004$, OR $[95 \% \mathrm{Cl}]=1.66[1.21,2.76])$, increased recanalization rate $(88$ vs. $60 \%, p<0.00001$, OR $[95 \%$
\end{abstract}


$\mathrm{Cl}]=3.99[2.73,5.84])$, and decreased mortality (26 vs. $41 \%, p=0.0004, \mathrm{OR}[95 \% \mathrm{Cl}]=1.86$ $[1.33,2.61])$, as well as an equal rate of $\mathrm{SICH}(5$ vs. $7 \%, p=0.15$, OR $[95 \% \mathrm{CI}]=1.68[0.82,3.43])$, in patients treated with SRT compared to those treated with IVT alone. Conclusions: The data from this meta-analysis suggest a possible superiority of SRT over IVT, pending positive results of randomized controlled trials. According to international recommendations, patients with BAO should preferentially be treated with SRT; if no contraindications exist, IVT should not be withheld.

\section{Introduction}

Acute basilar artery occlusion (BAO) is a rare condition, accounting for only about $3 \%$ of all ischemic strokes [1]. Untreated, it is associated with $>90 \%$ mortality and a high level of dependency among survivors [2,3]. The primary goal of acute therapy is to reestablish blood flow in the basilar artery, which can be achieved through intravenous application of alteplase (intravenous thrombolysis [IVT]) or endovascular disruption of the thrombus (endovascular therapy [EVT]). Although there is a historically grown trend towards EVT [4], there is no good evidence that supports the assumption that EVT is superior to IVT. A previous meta-analysis failed to demonstrate a benefit in clinical outcome with EVT versus IVT [5]. However, EVT has substantially developed in recent years, and recanalization rates have dramatically improved. The advent of stent retrievers marked the current standard for stroke treatment in the anterior circulation, with recanalization rates of $\geq 80 \%[6,7]$.

The positive results of recent thrombectomy trials prompted us to perform this updated review, since a growing number of studies on BAO have been published. In this systematic review and meta-analysis, we want to summarize and compare the treatment of BAO with IVT and stent retriever thrombectomy (SRT). We hypothesized that SRT would not only increase recanalization rates but also improve clinical outcome.

\section{Materials and Methods}

\section{Search Strategy and Data Collection}

Using PubMed, we identified studies (published in final form) through June 2018 reporting on patients $>18$ years of age with acute BAO treated with SRT or IVT with alteplase as first-line treatment. IVT prior to SRT was allowed. Any additional treatment was allowed, but if it was applied in $>50 \%$ of the patients in a study, the study was excluded. Both prospective and retrospective designs were allowed. Only studies reporting on $\geq 15$ patients were included. If study groups published successive articles with accumulating numbers of patients, only the most recent publication was included. Stroke registries from which information on patients with acute BAO and/or outcome and/or specific therapeutic approach could not be parsed were excluded. Review articles, (conference) abstracts, experimental or animal studies, and articles lacking radiological or clinical data were excluded. As minimum information, data on (1) recanalization rate, (2) clinical outcome, and (3) mortality were required. Favorable outcome was defined as a score of $0-2$ on the modified Rankin Scale (mRS) at a follow-up after discharge. Successful recanalization was defined as TICI (Thrombolysis in Cerebral Infarction) grades $2 \mathrm{~b}-3$. Some older studies did not adhere to these contemporary definitions of favorable outcome and successful recanalization. In these instances, the study definitions of favorable outcome and successful recanalization were kept, but this was explicitly stated. 


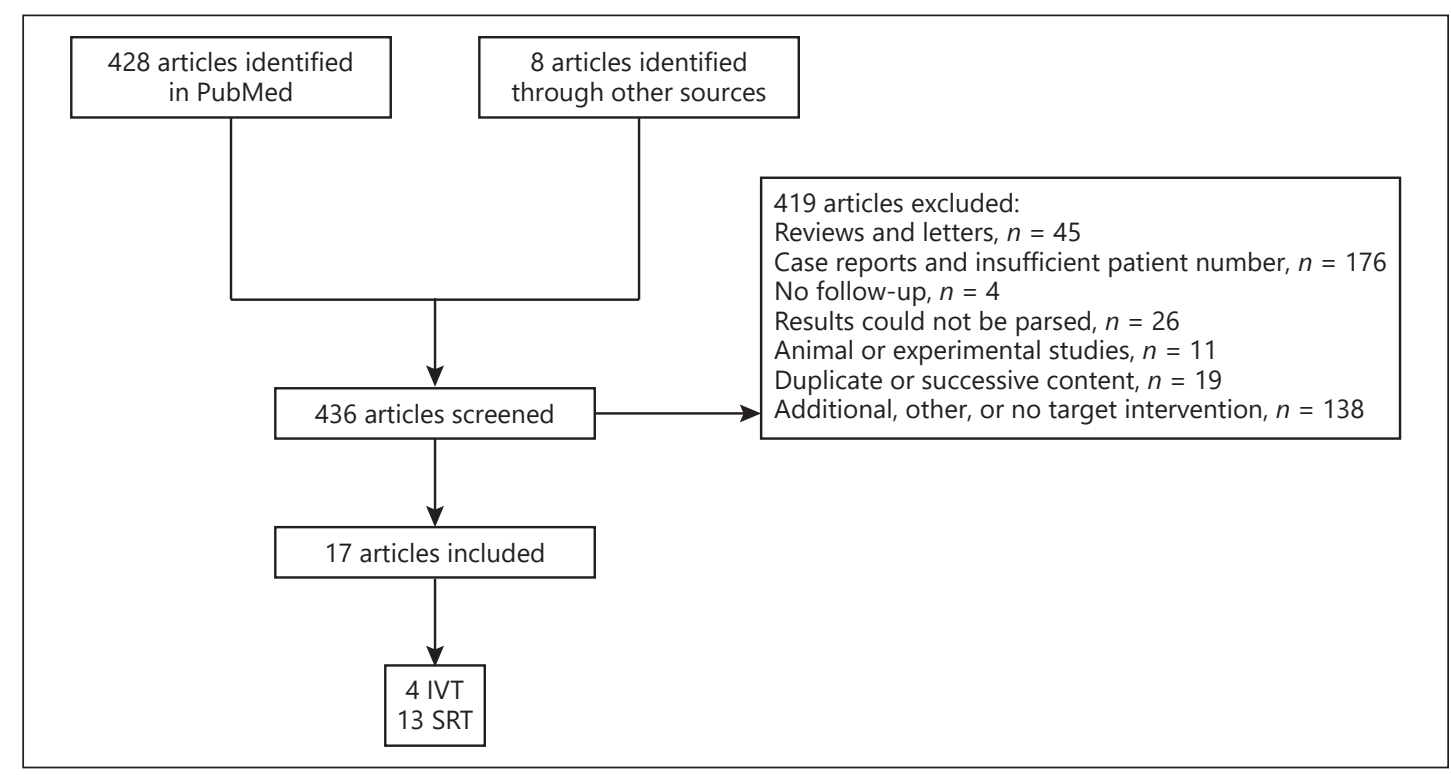

Fig. 1. Data collection. IVT, intravenous thrombolysis; SRT, stent retriever thrombectomy.

Two investigators (one board-certified radiologist and one board-certified neurologist) reviewed the studies and performed additional handsearching, e.g. of the literature sections of retrieved articles. If necessary, authors were contacted to obtain missing data. Data pertaining to the study design (study period, country where the study was conducted, and inclusion design), patient demographics (number of patients, age, and sex), and treatmentrelated information (treatment modality and additional treatment) were extracted and tabulated. Data pertaining to outcome parameters (recanalization rate, clinical outcome, mortality, and symptomatic intracranial hemorrhage [SICH]) were also extracted and tabulated. Subsequently, events, nonevents, and total numbers of cases in the respective category were extracted, calculated, and tabulated for each individual study and for the combined study population. Disagreement was resolved in consensus.

\section{Statistical Analysis}

Odds ratios (ORs) were calculated using Mantel-Haenszel risk ratio estimation. Results are given as OR and the $95 \%$ confidence interval (95\% CI). The $\chi^{2}$ test was used to compare the outcome parameters clinical outcome, recanalization, mortality, and SICH. $I^{2}$ statistics was used to express heterogeneity. The level of significance was set at $p<0.05$. Continuous data are reported as mean \pm SD or median and range, categorical data as frequencies and percentages where appropriate. All calculations were made using Review Manager (version 5.3; The Cochrane Collaboration) and OpenMetaAnalyst (Tufts Medical Center, 2012).

\section{Results}

A total of 17 studies (4 on IVT and 13 on SRT) [8-24] with a total of 672 patients (IVT, $n=314$; SRT, $n=358$ ) were retrieved (see Fig. 1 for data collection). The data collection period ranged back to 1985 for the IVT group, while stent retrievers have only been used since 2009. The included studies had an overall preponderance of European centers. While in the IVT group no Asian studies were included, the SRT group included 6 Asian studies with 198 


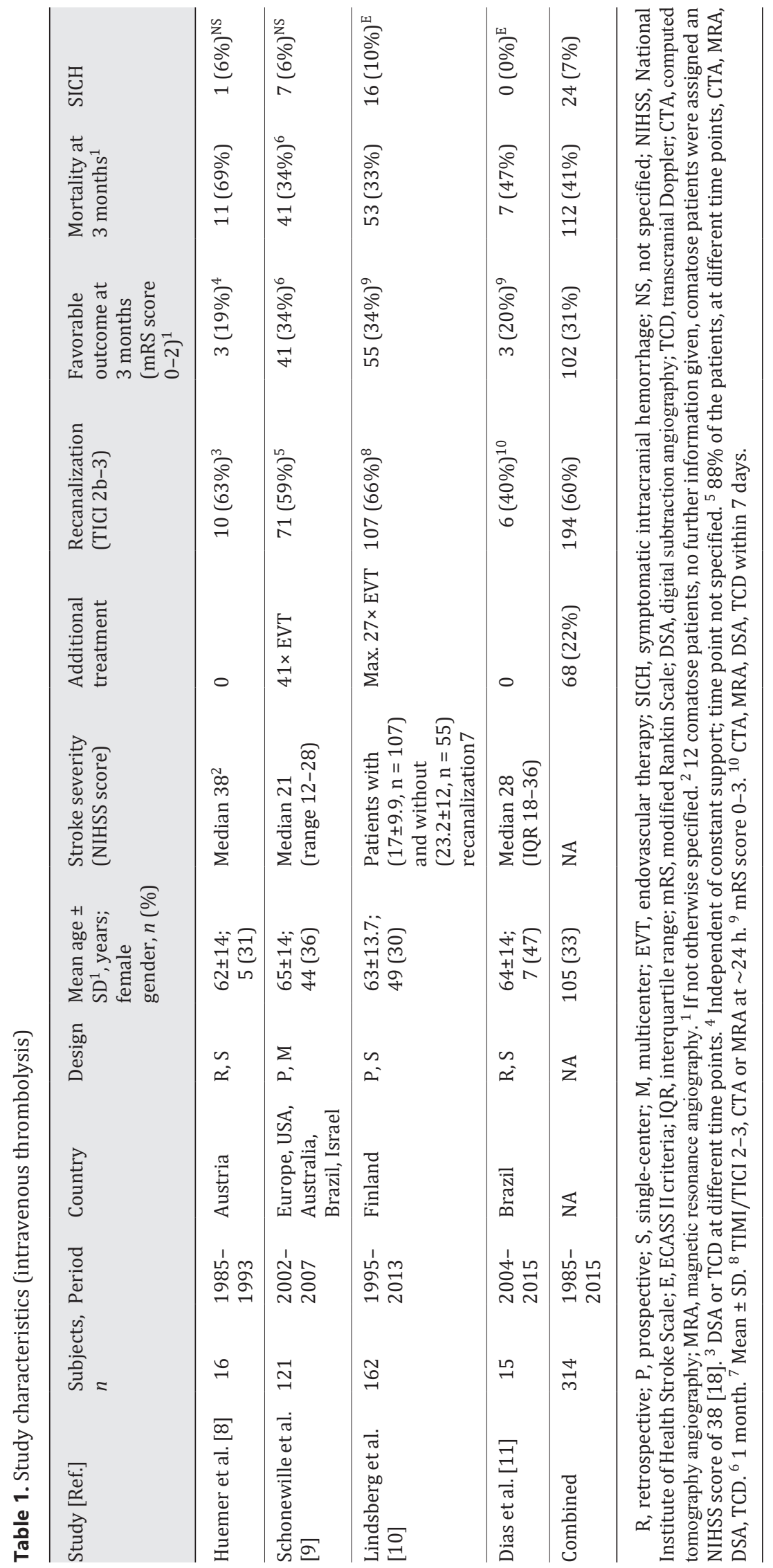


patients (53\%). Most of the studies were retrospective and single-center studies; however, 2 of the 4 IVT studies were prospective. In the SRT group, the Solitaire ${ }^{\circledR}$ and Trevo ${ }^{\circledR}$ devices were most often used. Additional endovascular treatment was performed in 59 cases $(16 \%)$. In the IVT group, additional treatment was performed in 68 cases (22\%). IVT was performed prior to SRT in 128 cases (34\%) (Tables 1,2).

Cumulatively, the target parameters "favorable clinical outcome" (43 vs. $31 \%, p=0.002$ ) and "successful recanalization" (88 vs. $60 \%, p<0.00001$ ) were highly significantly more frequently achieved in the SRT group. Mortality was highly significantly lower in the SRT group ( 22 vs. $41 \%, p=0.0003$ ). The occurrence of SICH was not statistically significantly different between the two groups ( 5 vs. $7 \%, p=0.15$ ) (Fig. 2).

Heterogeneity $\left(I^{2}\right)$ was $18 \%(p=0.301)$ for favorable clinical outcome, $37 \%(p=0.190)$ for successful recanalization, $69 \%(p=0.021)$ for mortality, and $0 \%(p=0.450)$ for SICH in the IVT group, while it was $40 \%(p=0.070), 64 \%(p<0.001), 58 \%(p=0.004)$, and $8 \%(p=$ $0.371)$, respectively, in the SRT group.

\section{Discussion}

In this meta-analysis, we found a significantly increased recanalization rate, an improved clinical outcome, decreased mortality, and an equal rate of SICH in patients treated with SRT compared to those treated with IVT alone.

\section{Treatment Modality}

The results are in line with positive results from recent trials of thrombectomy in the anterior circulation. However, they contradict the results of the BASICS registry [9], which could not demonstrate a superior efficacy of EVT over IVT. Further, a previous meta-analysis could not establish any superiority of either treatment modality [5]. Both studies, however, were performed or included studies before stent retrievers were used. Stent retrievers are currently regarded as the single most effective mechanical thrombectomy device. Technically, they are temporarily deployable stents migrating radially through the thrombus which can subsequently be extracted. They allow for high recanalization rates, as demonstrated in recent trials.

The main criticism of the BASICS registry pertaining to the question of this review is that of the 121 patients included in the IVT group, 41 had received additional EVT. Thus, the effects of the different treatment modalities could not be parsed. In the cohort presented here, $18 \%$ (127 of 688) of the patients received 1 additional treatment or 1 additional thrombectomy device was used, and 36\% (128 of 374) of the patients treated with SRT had received IVT prior to the intervention. By excluding studies that used $>1$ thrombectomy device in $>50 \%$ of the patients, we tried to reduce the effect of combined therapies.

\section{Clinical Outcome}

As the most important result, we found a higher rate of favorable outcomes as well as decreased mortality after SRT. An mRS score $\leq 2$ at 90 days is considered to represent functional independency and thus a favorable outcome. Although this threshold has not been validated and some authorities have questioned the adequacy of using this threshold for BAO [10, 11], we chose to adhere to this strict definition of favorable outcome. Had we chosen a less strict definition of favorable outcome, the result in favor of SRT would probably have been even more pronounced. Further, some patients $(n=152)$ were followed up at other time points. 


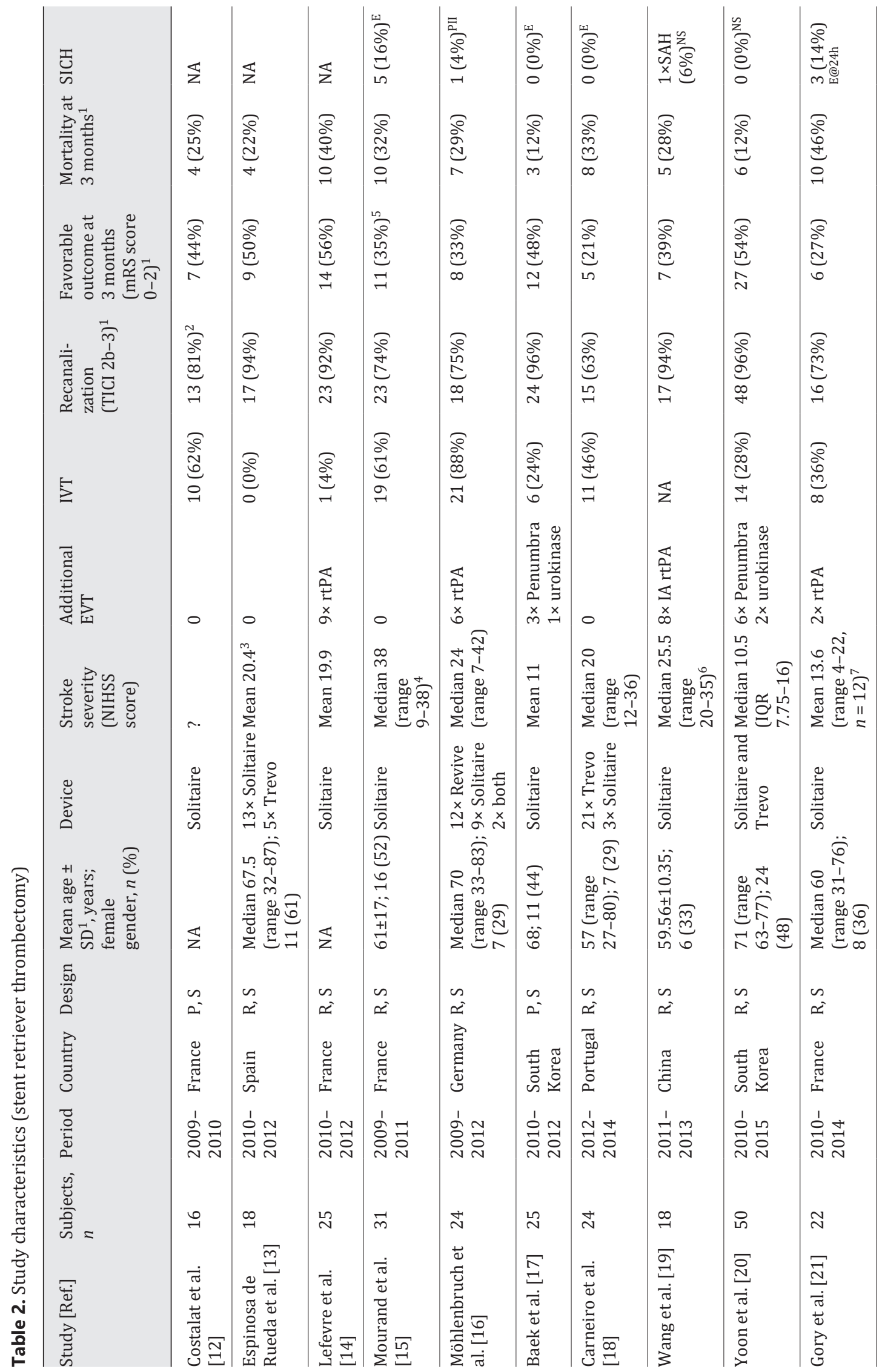




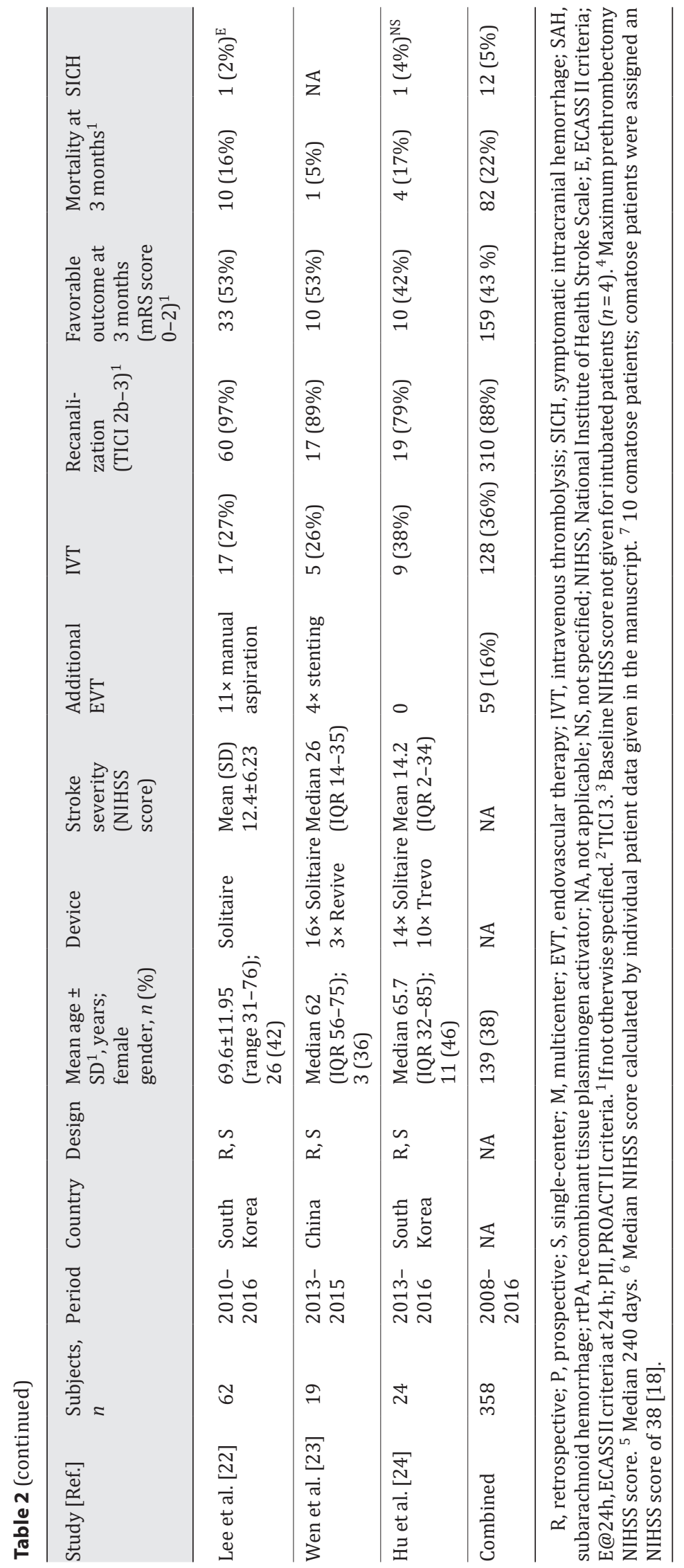




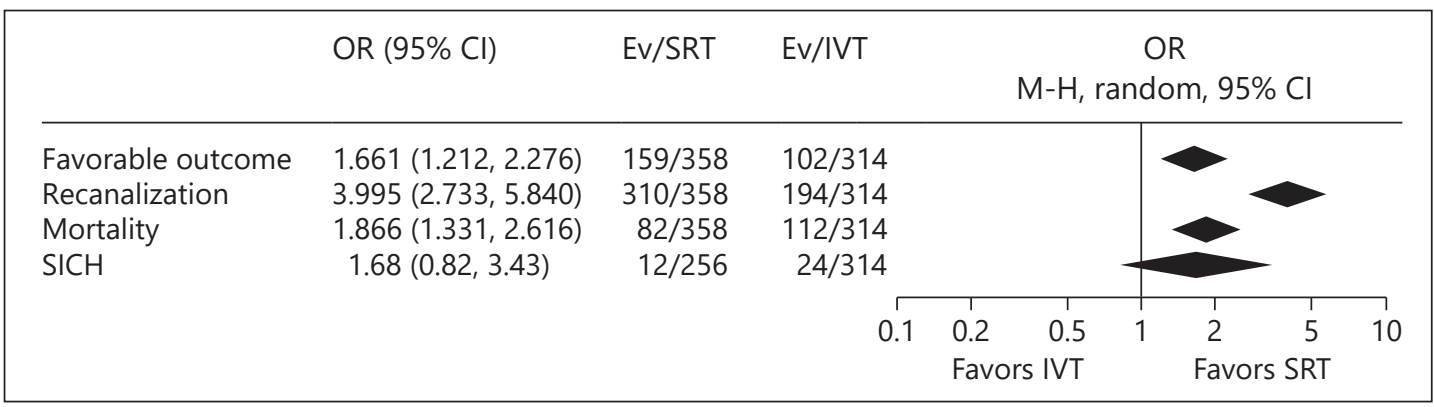

Fig. 2. Detailed results with forest plots. OR, odds ratio; CI, confidence interval; Ev, events; SRT, stent retriever thrombectomy; IVT, intravenous thrombolysis; SICH, symptomatic intracranial hemorrhage; M-H, Mantel-Haenszel.

\section{Recanalization}

We found a significantly higher recanalization rate among patients treated with SRT. This finding is not new, as a previous meta-analysis had demonstrated higher recanalization rates with EVT in general than with IVT. The success of recanalization with IVT was demonstrated to be dependent on thrombus length. Long thrombi were shown to be more resistant both in the anterior [25] and the posterior circulation [26]. In contrast, it was recently demonstrated that EVT is effective even when dealing with long thrombi $[27,28]$.

In patients treated with SRT, BAO was proven directly prior to therapy via the gold standard digital subtraction angiography. In contrast, many patients receiving IVT were diagnosed by magnetic resonance angiography, with the caveat of possible overestimation of a stenosis and misclassification as an occlusion. Conversely, demonstration of vessel patency in patients treated with SRT was immediately available. In contrast, patients treated with IVT were observed within undefined time spans after therapy by various imaging modalities.

It is often stated that imaging parameters should be used to select patients eligible for therapy. In summary, patients with extensive infarctions in the brain stem and the posterior territory are less likely to benefit from recanalization. Various techniques ranging from nonenhanced cranial computed tomography (CT) [29], CT angiography source imaging [30], and CT perfusion [31] to diffusion-weighted magnetic resonance imaging [32] have been used to identify infarcted tissue. However, recanalization itself has been identified as a condition without which only a minimal chance of favorable outcome is reported $[5,10]$.

\section{Symptomatic Intracranial Hemorrhage}

We found an equal rate of SICH in the two treatment groups. The overall rate of occurrence of SICH was low, so these results certainly have to be regarded with caution. In most of the studies, SICH was defined according to the ECASS II criteria. Various other definitions have been used, especially in the era preceding the ECASS. The incidence of SICH found here is in the order of magnitude of the incidence of SICH in IVT trials [33] and recent thrombectomy trials $[6,7]$.

There are several limitations to our results. The data presented here cannot replace a randomized controlled trial. Only a minority of the studies were designed prospectively, and none directly compared IVT and SRT. This introduces several possibilities for bias, especially heterogeneity in the baseline characteristics of the included subjects. Since data on baseline characteristics were either not given (e.g. medication), incomplete (missing data), or inconsistent (different measures of location), they are listed in Tables 1 and 2, but no reliable statistical comparison could be made. In the absence of controlled trials, it has to be stressed that heterogeneity in baseline characteristics is expected to be large. 
Furthermore, data on IVT only for BAO are limited, and few studies contribute the bulk of the patient cohort. Studies on the IVT cohort date back as far as 1985, which can be regarded as "historical." Although the majority of patients were included after 1995, an influence of major achievements in stroke medicine - such as greater availability of stroke units and changes in medical therapy such as statin use and dual platelet inhibition - on overall outcome cannot be excluded. Especially older studies did not report outcome parameters using scales which are in use today (the NIHSS for clinical symptoms at admission, the ECASS criteria for intracranial hemorrhage, and the mRS for functional outcome) and thus do not provide standardized, comparable results.

We provide comprehensive data on a comparison between IVT and latest-generation SRT. Previous meta-analyses have focused primarily on outcome parameters alone [34], compared intraarterial thrombolysis with mechanical thrombectomy [35], focused solely on SRT [21, 36], or compared IVT with EVT including intraarterial pharmacological thrombolysis and mechanical thrombectomy including first-generation devices or omitted older work $[5,10]$.

The results of randomized controlled trials currently conducted [37] are expected to shed light on this question still unanswered in stroke medicine. Recently, the results of the BEST trial (NCT 02441556) have been published as a conference abstract [38]. The BEST trial was designed as a multicenter prospective randomized controlled trial in China. The trial was terminated prematurely because of high crossover rates between the treatment groups. In conclusion, no superiority of thrombectomy over medical treatment could be demonstrated in the intention-to-treat analysis. However, the patients eventually treated with EVT fared better than the patients treated medically alone.

In conclusion, the data from this meta-analysis suggest a possible superiority of SRT over IVT, pending positive results of randomized controlled trials. Our results support the approach suggested by several international organizations [39]: if no contraindications exist, patients with acute BAO should receive IVT and be transferred to a neurovascular center, where mechanical thrombectomy preferably with stent retrievers can be performed.

\section{Acknowledgements}

We acknowledge financial support for publication fees by the state of Schleswig-Holstein within the funding program Open Access Publikationsfonds.

\section{Statement of Ethics}

The authors have no ethical conflicts to disclose.

\section{Disclosure Statement}

The authors have no conflicts of interest to disclose.

\section{References}

1 Weimar C, Goertler M, Harms L, Diener HC. Distribution and outcome of symptomatic stenoses and occlusions in patients with acute cerebral ischemia. Arch Neurol. 2006 Sep;63(9):1287-91.

2 Hacke W, Zeumer H, Ferbert A, Brückmann H, del Zoppo GJ. Intra-arterial thrombolytic therapy improves outcome in patients with acute vertebrobasilar occlusive disease. Stroke. 1988 Oct;19(10):1216-22. 


\begin{tabular}{l|l}
\hline \multicolumn{2}{l}{ Cerebrovasc Dis Extra 2019;9:46-56 } \\
\hline DOI: 10.1159/000499665 & $\begin{array}{l}\text { (c) 2019 S. Karger AG, Basel } \\
\text { www.karger.com/cee }\end{array}$ \\
\hline
\end{tabular}

Shu et al.: SRT versus IVT in Acute Basilar Occlusion

3 Brandt T, von Kummer R, Müller-Küppers M, Hacke W. Thrombolytic therapy of acute basilar artery occlusion. Variables affecting recanalization and outcome. Stroke. 1996 May;27(5):875-81.

4 Zeumer H, Hacke W, Kolmann HL, Poeck K. Lokale Fibrinolysetherapie bei Basilaris-Thrombose. Dtsch Med Wochenschr. 1982 May;107(19):728-31.

5 Lindsberg PJ, Mattle HP. Therapy of basilar artery occlusion: a systematic analysis comparing intra-arterial and intravenous thrombolysis. Stroke. 2006 Mar;37(3):922-8.

6 Berkhemer OA, Fransen PS, Beumer D, van den Berg LA, Lingsma HF, Yoo AJ, et al.; MR CLEAN Investigators. A randomized trial of intraarterial treatment for acute ischemic stroke. N Engl J Med. 2015 Jan;372(1):11-20.

7 Saver JL, Goyal M, Bonafe A, Diener HC, Levy EI, Pereira VM, et al.; SWIFT PRIME Investigators. Stent-retriever thrombectomy after intravenous t-PA vs. t-PA alone in stroke. N Engl J Med. 2015 Jun;372(24):2285-95.

8 Huemer M, Niederwieser V, Ladurner G. Thrombolytic treatment for acute occlusion of the basilar artery. J Neurol Neurosurg Psychiatry. 1995 Feb;58(2):227-8.

9 Schonewille WJ, Wijman CA, Michel P, Rueckert CM, Weimar C, Mattle HP, et al.; BASICS study group. Treatment and outcomes of acute basilar artery occlusion in the Basilar Artery International Cooperation Study (BASICS): a prospective registry study. Lancet Neurol. 2009 Aug;8(8):724-30.

10 Lindsberg PJ, Sairanen T, Nagel S, Salonen O, Silvennoinen H, Strbian D. Recanalization treatments in basilar artery occlusion - systematic analysis. Eur Stroke J. 2016 Mar;1(1):41-50.

11 Dias FA, Alessio-Alves FF, Castro-Afonso LH, Cougo PT, Barreira CM, Camilo MR, et al. Clinical Outcomes of Patients with Acute Basilar Artery Occlusion in Brazil: An Observational Study. J Stroke Cerebrovasc Dis. 2017 Oct;26(10):2191-8.

12 Costalat V, Machi P, Lobotesis K, Maldonado I, Vendrell JF, Riquelme C, et al. Rescue, combined, and standalone thrombectomy in the management of large vessel occlusion stroke using the Solitaire device: a prospective 50-patient single-center study: timing, safety, and efficacy. Stroke. 2011 Jul;42(7):1929-35.

13 Espinosa de Rueda M, Parrilla G, Zamarro J, García-Villalba B, Hernández F, Moreno A. Treatment of acute vertebrobasilar occlusion using thrombectomy with stent retrievers: initial experience with 18 patients. AJNR Am J Neuroradiol. 2013 May;34(5):1044-8.

14 Lefevre PH, Lainay C, Thouant P, Chavent A, Kazemi A, Ricolfi F. Solitaire FR as a first-line device in acute intracerebral occlusion: a single-centre retrospective analysis. J Neuroradiol. 2014 Mar;41(1):80-6.

15 Mourand I, Machi P, Milhaud D, Picot MC, Lobotesis K, Arquizan C, et al. Mechanical thrombectomy with the Solitaire device in acute basilar artery occlusion. J Neurointerv Surg. 2014 Apr;6(3):200-4.

16 Möhlenbruch M, Stampfl S, Behrens L, Herweh C, Rohde S, Bendszus M, et al. Mechanical thrombectomy with stent retrievers in acute basilar artery occlusion. AJNR Am J Neuroradiol. 2014 May;35(5):959-64.

17 Baek JM, Yoon W, Kim SK, Jung MY, Park MS, Kim JT, et al. Acute basilar artery occlusion: outcome of mechanical thrombectomy with Solitaire stent within 8 hours of stroke onset. AJNR Am J Neuroradiol. 2014 May;35(5): 989-93.

18 Carneiro AA, Rodrigues JT, Pereira JP, Alves JV, Xavier JA. Mechanical thrombectomy in patients with acute basilar occlusion using stent retrievers. Interv Neuroradiol. 2015 Dec;21(6):710-4.

19 Wang L, Shi W, Su Z, Liu X, Su H, Liu J, et al. Endovascular treatment of severe acute basilar artery occlusion. J Clin Neurosci. 2015 Jan;22(1):195-8.

20 Yoon W, Kim SK, Heo TW, Baek BH, Lee YY, Kang HK. Predictors of Good Outcome After Stent-Retriever Thrombectomy in Acute Basilar Artery Occlusion. Stroke. 2015 Oct;46(10):2972-5.

21 Gory B, Eldesouky I, Sivan-Hoffmann R, Rabilloud M, Ong E, Riva R, et al. Outcomes of stent retriever thrombectomy in basilar artery occlusion: an observational study and systematic review. J Neurol Neurosurg Psychiatry. 2016 May;87(5):520-5.

22 Lee YY, Yoon W, Kim SK, Baek BH, Kim GS, Kim JT, et al. Acute Basilar Artery Occlusion: Differences in Characteristics and Outcomes after Endovascular Therapy between Patients with and without Underlying Severe Atherosclerotic Stenosis. AJNR Am J Neuroradiol. 2017 Aug;38(8):1600-4.

23 Wen WL, Li ZF, Zhang YW, Yang PF, Simfukwe K, Fang YB, et al. Effect of Baseline Characteristics on the Outcome of Stent Retriever-Based Thrombectomy in Acute Basilar Artery Occlusions: A Single-Center Experience and Pooled Data Analysis. World Neurosurg. 2017 Aug;104:1-8.

24 Hu SY, Yi HJ, Lee DH, Hong JT, Sung JH, Lee SW. Effectiveness and Safety of Mechanical Thrombectomy with Stent Retrievers in Basilar Artery Occlusion: Comparison with Anterior Circulation Occlusions. J Korean Neurosurg Soc. 2017 Nov;60(6):635-43.

25 Riedel CH, Zimmermann P, Jensen-Kondering U, Stingele R, Deuschl G, Jansen O. The importance of size: successful recanalization by intravenous thrombolysis in acute anterior stroke depends on thrombus length. Stroke. 2011 Jun;42(6):1775-7.

26 Strbian D, Sairanen T, Silvennoinen H, Salonen O, Lindsberg PJ. Intravenous thrombolysis of basilar artery occlusion: thrombus length versus recanalization success. Stroke. 2014 Jun;45(6):1733-8.

27 Seker F, Pfaff J, Wolf M, Schönenberger S, Nagel S, Herweh C, et al. Impact of thrombus length on recanalization and clinical outcome following mechanical thrombectomy in acute ischemic stroke. J Neurointerv Surg. 2017 Oct; 9(10):937-9.

28 Shu L, Riedel C, Meyne J, Jansen 0, Jensen-Kondering U. Successful recanalization in acute basilar artery occlusion treated with endovascular therapy is independent of thrombus length. J Neurointerv Surg. 2017 Nov; 9(11):1047-52. 
29 Strbian D, Sairanen T, Silvennoinen H, Salonen O, Kaste M, Lindsberg PJ. Thrombolysis of basilar artery occlusion: impact of baseline ischemia and time. Ann Neurol. 2013 Jun;73(6):688-94.

30 Puetz V, Sylaja PN, Coutts SB, Hill MD, Dzialowski I, Mueller P, et al. Extent of hypoattenuation on CT angiography source images predicts functional outcome in patients with basilar artery occlusion. Stroke. 2008 Sep; 39(9):2485-90.

31 Pallesen LP, Gerber J, Dzialowski I, van der Hoeven EJ, Michel P, Pfefferkorn T, et al.; BASICS Study Group. Diagnostic and Prognostic Impact of pc-ASPECTS Applied to Perfusion CT in the Basilar Artery International Cooperation Study. J Neuroimaging. 2015 May-Jun;25(3):384-9.

32 Nagel S, Herweh C, Köhrmann M, Huttner HB, Poli S, Hartmann M, et al. MRI in patients with acute basilar artery occlusion - DWI lesion scoring is an independent predictor of outcome. Int J Stroke. 2012 Jun; 7(4): 282-8.

33 Hacke W, Kaste M, Fieschi C, von Kummer R, Davalos A, Meier D, et al.; Second European-Australasian Acute Stroke Study Investigators. Randomised double-blind placebo-controlled trial of thrombolytic therapy with intravenous alteplase in acute ischaemic stroke (ECASS II). Lancet. 1998 Oct;352(9136):1245-51.

34 Kumar G, Shahripour RB, Alexandrov AV. Recanalization of acute basilar artery occlusion improves outcomes: a meta-analysis. J Neurointerv Surg. 2015 Dec;7(12):868-74.

35 Mak CH, Ho JW, Chan KY, Poon WS, Wong GK. Intra-arterial revascularization therapy for basilar artery occlusion - a systematic review and analysis. Neurosurg Rev. 2016 Oct;39(4):575-80.

36 Phan K, Phan S, Huo YR, Jia F, Mortimer A. Outcomes of endovascular treatment of basilar artery occlusion in the stent retriever era: a systematic review and meta-analysis. J Neurointerv Surg. 2016 Nov;8(11):1107-15.

37 van der Hoeven EJ, Schonewille WJ, Vos JA, Algra A, Audebert HJ, Berge E, et al.; BASICS Study Group. The Basilar Artery International Cooperation Study (BASICS): study protocol for a randomised controlled trial. Trials. 2013 Jul;14(1):200.

38 Liu X, Xu G, Zi W, Liu W, Zhou Z, Shi Z, et al. Basilar artery occlusion endovascular intervention versus standard medical treatment (BEST) trial: primary results of a multicentre randomized controlled trial. Int J Stroke. 2018;13 2S:227.

39 Wahlgren N, Moreira T, Michel P, Steiner T, Jansen O, Cognard C, et al.; ESO-KSU, ESO, ESMINT, ESNR and EAN. Mechanical thrombectomy in acute ischemic stroke: Consensus statement by ESO-Karolinska Stroke Update 2014/2015, supported by ESO, ESMINT, ESNR and EAN. Int J Stroke. 2016 Jan;11(1):134-47. 